\title{
Infecciones respiratorias virales en pacientes hospitalizados en una unidad de cuidados intensivos neonatales
}

\author{
Viral respiratory infections in hospitalized patients in a \\ neonatal intensive care unit
}

\author{
Patricia Arias ${ }^{1}$, Rosanna Fonseca ${ }^{1}$, Chantal Real ${ }^{1}$, Leticia Zapata ${ }^{1}$, Larissa Genes ${ }^{1}$, Raquel \\ Escobar $^{2}$, Elke Strübing ${ }^{2}$
}

\section{RESUMEN}

Introducción: Las infecciones respiratorias virales constituyen una problemática nacional y las unidades de cuidados intensivos neonatales, no están exentas de estas. Objetivo: Describir la frecuencia y características clínicas de las infecciones respiratorias virales en neonatos internados en un servicio de Neonatología durante el periodo de enero 2017 a octubre 2018. Materiales y Métodos: Estudio observacional, descriptivo, retrospectivo. Fueron analizadas historias clínicas de pacientes hospitalizados en la unidad de cuidados intensivos neonatales, que tuvieron diagnóstico de infecciones respiratorias de origen viral, el método de diagnóstico fue inmunofluorescencia directa, inmunocromatografía y PCR en tiempo real. Se excluyeron pacientes que procedan de otro servicio $y$ hayan iniciado los síntomas antes del ingreso a nuestro centro. Variables: edad gestacional, edad posnatal, peso de nacimiento, síntomas, tipo de virus, prescripción de antibióticos. Los datos fueron analizados con Excel, utilizando estadísticas descriptivas. Resultados: De los 761 pacientes, 65 pacientes presentaron diagnóstico de infección respiratoria viral: frecuencia del 8,5\%. El promedio de edad fue de $17 \pm 10$ días El método de diagnóstico más frecuente fue inmunofluorescencia $92 \%$, Los síntomas más comunes fueron las secreciones respiratorias (74\%) y desaturaciones $(64 \%)$; Los virus más frecuentes fueron: Sincitial Respiratorio 26\%, Influenza B $25 \%$, Influenza A en un $18 \%$. El $66 \%$ requirió tratamiento de soporte respiratorio. Un paciente falleció por causas no relacionadas a la infección respiratoria viral. Conclusiones: La frecuencia de infecciones virales fue del $8,5 \%$. Los virus más frecuentes fueron VSR e influenza B.

\section{ABSTRACT}

Introduction: Viral respiratory infections are a national problem and neonatal intensive care units are not exempt from these infections. Objective: To describe the frequency and clinical characteristics of viral respiratory infections in newborns admitted to a Neonatology unit during january 2017 to October 2018 timeframe. Materials and Methods: This was an observational, descriptive and retrospective study. We reviewed the clinical records of patients admitted to the neonatal intensive care unit who were also diagnosed with a viral respiratory infection. To determine the etiology, we used direct immunofluorescence, immunochromatography and realtime PCR. Patients who were transferred from another service and whose symptoms began prior to transfer to our unit were excluded. Variables: gestational age, postnatal age, birth weight, symptoms, type of virus, antibiotic prescription. Data were analyzed with Excel, using descriptive statistics. Results: Of the 761 patients, 65 patients were diagnosed with a viral respiratory infection, a frequency of $8.5 \%$. The average age was $17 \pm 10$ days. The most frequent diagnostic method was immunofluorescence (92\%). The most common symptoms were respiratory secretions $(74 \%)$ and desaturations (64\%); The most frequent viruses were: Respiratory Syncytial (26\%), Influenza B (25\%) and Influenza A (18\%). $66 \%$ required respiratory support treatment. One patient died from causes unrelated to the viral respiratory infection. Conclusions: The frequency of viral infections was $8.5 \%$. The most frequent viruses were RSV and influenza B. More than half needed respiratory support. No patient died.

${ }^{1}$ Servicio de Neonatología. Hospital Materno Infantil San Pablo, Asunción. Paraguay

${ }^{2}$ Departamento de Docencia. Hospital Materno Infantil San Pablo, Asunción. Paraguay

Correspondencia: Patricia Arias Correo: arias18405@gmail.com

Conflicto de Interés: Los autores declaran no poseer conflicto de interés

Recibido: 10/08/2019 Aceptado:6/11/2019

Doi: https://doi.org/10.31698/ped.46032019003

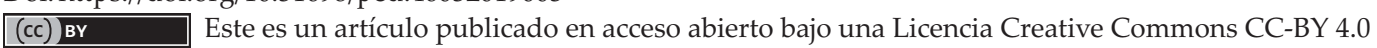


Más de la mitad necesitaron soporte respiratorio. Ningún paciente falleció.

Palabras clave: Recién nacido, prematuros, enfermedad viral, unidad de cuidados intensivos neonatales.

\section{INTRODUCCIÓN}

Las infecciones respiratorias agudas constituyen las enfermedades infecciosas más frecuentes; con significativa morbilidad y mortalidad durante la infancia. En tiempos pasados, todos los esfuerzos se centraban en el manejo de infecciones respiratorias bacterianas, hoy en día éstas han disminuido debido al mejor desarrollo de medidas preventivas como las vacunas, por lo que actualmente, sobre todo por el desarrollo de nuevos métodos de diagnóstico y mayor conocimiento sobre la existencia de infecciones virales, este tipo de enfermedades, están ganando importancia ${ }^{(1-5)}$.

Los recién nacidos, sobre todo los prematuros, son susceptibles a infecciones, debido a la inmadurez y la deficiencia de su sistema inmune, y a la exposición a procedimientos invasivos en las unidades neonatales. La respuesta innata de las citoquinas contra los virus en un recién nacido puede ser inadecuada o, por el contrario, exagerada, y asociada con incremento de la severidad de algunas enfermedades. Todos estos factores argumentan el elevado riesgo de infecciones ${ }^{(6)}$. Además, se plantea que los prematuros con déficit de surfactante están más predispuestos a infecciones respiratorias, ya que el surfactante actúa como barrera protegiendo la superficie alveolar de agresiones ${ }^{(7)}$.

Los virus que con mayor frecuencia se asocian a las infecciones del tracto respiratorio en unidades de neonatología son el virus sincitial respiratorio (VSR), el rinovirus, los virus parainfluenza, influenza y el adenovirus ${ }^{(8)}$. Aún no se conoce con detalle el papel que desempeñan en las terapias intensivas neonatales, pero algunos, están asociados a descompensación clínica, como aparición de apneas, fiebre, letargia y rechazo alimentario, llegando a necesitar soporte respiratorio e incluso en casos más severos, oxigenación por membrana
Keywords: Newborn, premature, viral disease, neonatal intensive care unit.

extracorpórea, sobre todo en los prematuros menores de 32 semanas o con peso inferior a 1.500 $\operatorname{gr}^{(3,6,9)}$.

Esto genera un inconveniente al momento de realizar el diagnóstico puesto que los casos graves, a veces, son indiferenciables de los causados por infecciones bacterianas $u$ otras enfermedades asociadas a la prematuridad, como la enterocolitis, la sepsis nosocomial o la apnea de la prematuridad, en este tipo de situaciones, la etiología viral se plantea en contadas ocasiones, lo que hace que no solamos considerar los virus respiratorios como agentes causales ${ }^{(9,10)}$.

La identificación del virus mediante el estudio genético constituyó un avance en el diagnóstico y manejo de las infecciones respiratorias virales. La introducción de la Reacción en cadena de la polimerasa (PCR), con sus diferentes técnicas moleculares, en tiempo real y las rápidas, permitió que nuevos virus sean identificados, y así el conocimiento de las infecciones respiratorias virales haya aumentado de forma importante ${ }^{(6,11)}$.

En la actualidad, existe tratamiento antiviral accesible en nuestro medio, para los virus de la Influenza. Existe además otro antiviral llamado cidofovir, para infecciones por adenovirus, no existen aún muchos estudios en pacientes pediátricos y neonatos, aunque ya fue aprobado por la FDA en el año 1996 para uso en pacientes inmunocomprometidos que padecen de infección grave por Adenovirus. Debido a esto, el pilar del manejo es el cuidado de soporte, tratamiento sintomático ${ }^{(4,6)}$.

En el marco de los cuidados hospitalarios, la detección de infecciones virales, son resultados de búsquedas activas. El objetivo del presente trabajo, 
fue determinar la frecuencia, características clínicas y evolución de las infecciones respiratorias de origen viral en neonatos hospitalizados en la unidad de cuidados intensivos neonatales.

\section{MATERIALES Y MÉTODOS}

Se realizó un estudio observacional descriptivo transversal y retrospectivo. Por medio de un muestreo consecutivo, se incluyó a recién nacidos con estadía en la UCIN de al menos 5 días o más y que tuvieron el diagnóstico de infección respiratoria aguda viral, por estudio de secreción nasofaríngea utilizando las técnicas de inmunofluorescencia (IFI), inmunocromatografía o PCR en tiempo real. Se excluyeron pacientes que hayan ingresado de otro servicio e iniciado los síntomas antes del ingreso. Las variables estudiadas fueron las demográficas, peso de nacimiento, edad gestacional, edad en el momento del diagnóstico, antecedentes patológicos, mes del año, tipo de test diagnóstico utilizado, síntomas, necesidad de asistencia respiratoria, sospecha de contacto, tratamiento recibido, complicaciones y factores asociados a la misma. Los datos fueron registrados en una planilla electrónica Excel, y para el análisis de datos se empleó el programa estadístico Epi Info 7.

Se respetó el derecho de confidencialidad de los datos, ya que para identificar a los pacientes se emplearon códigos numéricos. Este trabajo no requirió de consentimiento informado pues se trabajó con expedientes médicos.

\section{RESULTADOS}

De un total de 761 ingresos de pacientes en la UCIN en el periodo de estudio, 65 pacientes llenaron los criterios de inclusión, Fueron 39 pacientes del año 2017 y 26 pacientes del periodo enero a octubre del año 2018. La prevalencia de infección respiratoria viral en la población estudiada fue de $8,5 \%$. Las características demográficas se encuentran en la Tabla 1.

Tabla 1. Características demográficas de los pacientes con infección viral hospitalizados en el Hospital materno infantil San Pablo N=65.

\begin{tabular}{lc}
\hline & $\mathbf{N}(\%)$ \\
\hline Sexo & $36(55,4)$ \\
Masculino & $29(44,6)$ \\
Femenino & $59(91)$ \\
\hline Prematurez & \\
\hline Peso de nacimiento & $26(40)$ \\
$\geq \mathbf{1 5 0 0 g}$ & $27(42)$ \\
$\mathbf{1 0 0 0 - 1 4 9 9}$ & $12(18)$ \\
$<\mathbf{1 0 0 0}$ & $17 \pm 10$ \\
\hline Edad al diagnóstico (días) & \\
Media DE &
\end{tabular}

Como antecedentes patológicos previos a la infección respiratoria viral, los pacientes presentaron: ductus arterioso permeable en 43 pacientes $(66 \%)$, requirieron surfactante en las primeras horas de vida 24 pacientes $(37 \%)$, SALAM 2 pacientes $(3 \%)$ y de broncodisplasia pulmonar previa a la infección 1 paciente. Ningún paciente tuvo cardiopatía compleja. Además 39 pacientes $(60 \%)$ presentaron algún grado de hipertensión pulmonar determinado por ecocardiografía Doppler. Los síntomas clínicos presentados por los pacientes se encuentran en la Tabla 2. 
Tabla 2. Síntomas presentados por los pacientes con infección respiratoria viral hospitalizados en el Hospital Materno Infantil San Pablo N =65.

\begin{tabular}{lc}
\hline Síntomas & Pacientes \\
\hline Secreciones nasales & $\mathbf{( N )}(\%)$ \\
Desaturaciones & $48(74)$ \\
Taquicardia & $42(64)$ \\
Dificultad respiratoria & $37(57)$ \\
Febrícula & $36(55)$ \\
Reticulado (piel) & $36(55)$ \\
Estornudos & $24(37)$ \\
Irritabilidad & $19(29)$ \\
Distensión abdominal & $17(27)$ \\
Apneas & $17(27)$ \\
Débito por sonda alimentación & $14(21)$ \\
Vómitos & $13(20)$ \\
Fiebre & $13(20)$ \\
Hipotermia & $11(17)$ \\
Enterocolitis Necrotizante & $2(3)$ \\
\hline
\end{tabular}

En 6 pacientes solo se constataron taquicardia, irritabilidad y febrícula, sin síntomas respiratorios. La búsqueda activa se realizó debido al nexo epidemiológico.
El manejo clínico respiratorio y los datos laboratoriales y de imágenes de los pacientes se encuentra en la Tabla 3.

Tabla 3. Manejo clínico, datos laboratoriales de los pacientes con infección respiratoria viral N=65.

\begin{tabular}{ll}
\hline FiO2 $(\%)$ & $\mathbf{2 8} \pm \mathbf{5}$ \\
Media DE & $\mathrm{N}(\%)$ \\
Oxigenoterapia & $43(66)$ \\
\hline $\begin{array}{l}\text { Soporte respiratorio } \\
\text { (ARM o CPAP) }\end{array}$ \\
\hline $\begin{array}{l}\text { Radiografía de tórax: } \\
\text { Infiltrado pulmonar intersticial }\end{array}$ \\
\hline $\begin{array}{l}\text { Hemograma: } \\
\text { Leucocitos normal }\end{array}$ \\
Leucocitos aumentados & $48(74)$ \\
\hline $\begin{array}{l}\text { Proteína C reactiva } \\
\text { Positiva }\end{array}$ & $40(61,5)$ \\
\hline
\end{tabular}

E1 $60 \%(26 / 65)$ de los pacientes recibieron oxigenoterapia por menos de 15 días. El diagnóstico se realizó por el estudio del hisopado nasofaríngeo, utilizando el test de inmunofluorescencia directa a
60 pacientes (92\%), inmunocromatografía a $3(5 \%)$ y PCR en tiempo real a 2 pacientes (3\%). Los gérmenes más frecuentemente aislados se encuentran en la Tabla 4. 
Tabla 4. Virus aislados en las muestras de hisopado de los pacientes con infección respiratoria (n=65).

\begin{tabular}{lcc}
\hline & N & $\mathbf{( \% )}$ \\
\hline VSR & 17 & 26 \\
Influenza B & 16 & 24 \\
Influenza & 12 & 18 \\
Adenovirus & 8 & 12 \\
Parainfluenza 2 & 4 & 6 \\
Methapneumovirus & 3 & 5 \\
Parainfluenza 3 & 3 & 5 \\
Influenza A y B & 1 & 2 \\
Rinovirus & 1 & 2 \\
\hline Total & $\mathbf{6 5}$ & $\mathbf{1 0 0}$ \\
\hline
\end{tabular}

Se detectó contacto en 40 pacientes (61,5\%). Estos fueron padres, personal de salud con sospecha de infección y otros pacientes con infecciones respiratorias virales confirmadas (epidemiología positiva). La mayoría de los contactos que no eran pacientes internados no contaban con diagnóstico laboratorial de infección.

Se tomaron hemocultivos en 27 pacientes $(41,5 \%)$ por sospecha de infección bacteriana asociada a cuidados de la salud (IACS) de los cuales en el 66,6\% (18/27) se inició tratamiento antibiótico. Retornaron positivos 2/27. De los 25 hemocultivos negativos se suspendió los antibióticos a 10 de los 18 pacientes con antibióticos (55,5\%), teniendo en cuenta la asociación de síntomas al cuadro respiratorio viral ya diagnosticado. En cuanto a los restantes 6 pacientes que continuaron con antibióticos, fue debido a sepsis clínica. Considerando la población ingresada al estudio, con sospecha de infección bacteriana y con hemocultivo negativo, el 22,2\% (6/27) recibieron esquema completo de antibióticos. El 58,5\% (38/65) no se consideró infección bacteriana por consiguiente no se tomaron hemocultivos. El esquema antibiótico más utilizado fue la combinación de Meropenem, Vancomicina y Amikacina, seguido por Cefotaxima y Vancomicina.

Tratamiento antiviral se utilizó en 26 pacientes $(40 \%)$, que consistió en Oseltamivir, con duración del tratamiento en promedio de $5 \pm 0,7$ días. La duración total de aislamiento arrojó una mediana de 14 días, mínimo 5 días y máximo 15 días. Esto varió según el germen aislado.

Entre las complicaciones se encontraron las atelectasias en 6 pacientes (11,1\%), neumonías en 13 pacientes $(24 \%)$. Estas se presentaron con más frecuencia en la infección por el virus de la influenza B y el VSR. Requirieron además asistencia respiratoria (ARM o CPAP).

Las complicaciones no se asociaron a factores de riesgo conocidos Tabla 5.

Tabla 5. Relación entre características de los recién nacidos y la aparición de complicaciones respiratorias.

\begin{tabular}{lcccc}
\hline Variable & Con complicaciones & Sin complicaciones & OR (IC 95\%) & Valor de p* \\
\hline Prematurez & $30(50,8 \%)$ & $29(49 \%)$ & $1(0,1-5,5)$ & 1 \\
Peso menor a 1500 gr. & $22(55 \%)$ & $18(45 \%)$ & $1,5(0,5-4,2)$ & 0,38 \\
Ductus arterioso persistente & $5(45,4 \%)$ & $6(54,5 \%)$ & $0,7(0,2-2,7)$ & 0,65 \\
Sexo masculino & $20(55,5 \%)$ & $16(44,4 \%)$ & $1,5(0,5-4,1)$ & 0,38 \\
\hline
\end{tabular}

* Prueba chi ${ }^{2}$ 
De los 65 pacientes incluidos en el estudio, 1 paciente falleció.

\section{DISCUSIÓN}

La frecuencia de infecciones respiratorias virales encontradas fue de un $8,6 \%$, similar a otros estudios $^{(1)}$. En este estudio se observó que las infecciones respiratorias virales predominaron en la prematurez y el peso entre 1000 y 1500 gr. Esto se podría deber en parte a que, en este hospital, existe un elevado número de nacimientos prematuros por ser un nosocomio de referencia nacional, por lo tanto, la mayoría de los pacientes tiene mayor susceptibilidad a las infecciones virales ${ }^{(2,9)}$.

En la población de pretérminos la falta de desarrollo pulmonar podría explicar la mayor susceptibilidad a infecciones respiratorias ${ }^{(7,12,13)}$.

El promedio de días de vida al momento del diagnóstico fue de dos semanas, similar a reportados en otros estudios. El servicio donde se realizó el estudio, está abierto a padres y madres y a pesar de las ventajas conocidas del apego precoz, se sabe que los adultos pueden ser fuente de infección ${ }^{(9,14)}$. Desde el punto de vista inmunológico el contacto piel a piel y el cuidado centrado en la familia son factores positivos al disminuir el estrés y potenciar la maduración y estabilización de todos los sistemas, no sólo el neurológico, además de potenciar una interacción más estrecha y positiva entre padres e hijos, por lo que a favor del niño prematuro es importante protocolizar y tener un sistema riguroso para detectar adultos con virosis tanto en el personal de salud como en los padres y visitas (abuelos, hermanos). Se destaca a través de este trabajo, la importancia de la inmunización pasiva con palivisumab, contra el VSR, en pacientes de riesgo durante su estancia hospitalaria y su seguimiento posterior al alta y la vacunación contra el virus de la influenza, teniendo en cuenta el predominio también del virus de la Influenza A y B en el entorno del recién nacido, tanto familiar como personal de blanco.

Los meses del año en los cuales se presentaron las infecciones respiratorias fueron muy variables, según la bibliografía, predominan en meses invernales y otoñales ${ }^{(5)}$.
El test más utilizado fue el de la inmunofluorescencia directa, debido a la facilidad de acceso al estudio, menor costo que otros y rapidez de resultados ${ }^{(6,15)}$. La PCR en tiempo real, más sensible y específica para detección de virus respiratorios ${ }^{(16)}$ aún no es muy accesible en el ámbito público, que es donde se realizó este trabajo.

El síntoma respiratorio más frecuente que presentaron los pacientes fue la presencia de secreciones respiratorias, lo cual también es el principal motivo por el cual se sospecha infección viral respiratoria en la etapa neonatal; sin embargo, se resalta que casi un $30 \%$ de la población de estudio no poseía síntomas respiratorios, resaltándose así la importancia del análisis del nexo epidemiológico para establecer las sospechas de infecciones de vías aéreas superiores (IVAS). Además de estornudos, síntoma común de infecciones respiratorias, se presentaron también, desaturaciones y apneas, que son síntomas comunes a otras patologías neonatales. También fue frecuente la presencia de dificultad respiratoria en un poco más de la mitad de los pacientes, evidenciando la repercusión de una infección viral respiratoria.

Otros síntomas frecuentes fueron inespecíficos y comunes a otras patologías de la etapa neonatal, como las distermias, reticulado de piel, irritabilidad, trastornos gastrointestinales. Toda esta amplia gama de síntomas generalmente genera confusión diagnóstica, llevando a sospechar infección bacteriana, toma de hemocultivos e inicio de cobertura antibiótica de amplio espectro. Lo más difícil actualmente es diferenciar los síntomas de infecciones virales de los de sepsis neonatal bacteriana ${ }^{(2,5,6)}$.

Como antecedentes cardiológicos no se encontraron cardiopatías congénitas complejas, que predisponen a infecciones severas ${ }^{(17)}$, pero sí un elevado número de antecedentes de ductus arterioso permeable, que al producir hiperflujo pulmonar, favorece el daño pulmonar y la predisposición a infecciones respiratorias ${ }^{(14)}$. También se encontró que más de la mitad de los pacientes presentó algún grado de hipertensión pulmonar previo a la adquisición de la infección viral. Se plantea que las infecciones respiratorias, así como otras afecciones del recién nacido, producen de forma secundaria aumento de 
la presión pulmonar, en consecuencia, la hipertensión pulmonar podría acompañar el curso de una infección respiratoria viral y no ser precisamente la causa de éstas ${ }^{(18)}$.

Considerando el riesgo de las infecciones virales el servicio actualmente sigue normas de aislamiento según cada tipo específico virus, el cual se encuentran en las guías de la CDC ${ }^{(19)}$.

En relación al manejo de los pacientes, la mayoría requirió oxigenoterapia, un elevado número de pacientes requirió soporte más avanzado, es decir CPAP o ARM, sobre todo las infecciones a VSR e Influenza A y B. Las infecciones respiratorias virales en los recién nacidos, sobre todo los prematuros, pueden generar elevada morbilidad respiratoria ${ }^{(6)}$. El tratamiento farmacológico con oseltamivir se utilizó para los casos confirmados de influenza, el único germen con tratamiento antiviral disponible en nuestro medio ${ }^{(4,6)}$.

En los estudios radiológicos en la mayoría de los pacientes se observó el patrón de infiltrado pulmonar, hallazgo que no permite diferenciar una infección bacteriana de una viral, debido a que los patrones radiológicos tienen características similares ${ }^{(5,2)}$. Por otro lado, la interpretación radiológica es inespecífica y a veces subjetiva, dependiente del observador.

Con respecto a la analítica sanguínea, no hubo predominio de leucocitosis, pero se sabe que los métodos actuales no son suficientemente sensibles ni específicos ${ }^{(5)}$. Por otro lado, la proteína $C$ reactiva (PCR), en un bajo porcentaje resultó positivo. Los estudios relacionan el valor positivo, mayor a 37,4 $\mathrm{mg} / \mathrm{dl}$, de la PCR más con infección bacteriana, aunque se requiere más estudios de su utilidad en infecciones virales ${ }^{(21)}$.

En un poco más de un cuarto de los pacientes se inició tratamiento antibiótico, a pesar de la sospecha de infección viral, por la amplia gama de síntomas de esta patología que se superponen o que son compatibles con síntomas de infecciones bacterianas. Estas infecciones bacterianas concomitantes se encuentran descritas en la literatura, así como también el desafío que implican debido a su difícil distinción ${ }^{(6,22)}$.

La combinación de antibióticos utilizados fueron meropenem vancomicina y amikacina, los cuales se iniciaron ante sospecha IACS, para ampliar cobertura contra gérmenes intrahospitalarios, causantes de sepsis neonatal tardía grave. Debido a la posibilidad diagnóstica y a elevada mortalidad de la sepsis neonatal, esta debe ser considerada en muchos $\operatorname{casos}^{(6,9,10)}$.

No se encontró asociación entre factores de riesgo conocidos y complicaciones respiratorias tal como reportan algunos estudios ${ }^{(6)}$. Esto podría ser atribuido al escaso número de pacientes en cada estrato de riesgo.

Finalmente, se presentó solo un caso de óbito, al parecer no relacionado a infección respiratoria viral. El presente trabajo tiene limitaciones por ser una revisión retrospectiva, y con escaso número de pacientes. Sin embargo, aportan elementos que son útiles para demostrar la necesidad de ampliar el espectro diagnóstico pensando en un origen viral y no sólo en un origen bacteriano, lo cual es positivo, ayudando al médico a mejorar el manejo de los pacientes y evitar el uso indiscriminado de antibióticos, pues es bien conocido que esto aumenta la morbimortalidad, especialmente en prematuros.

\section{CONCLUSIONES}

Se encontró una frecuencia del $8.5 \%$ de cuadros respiratorios virales. El promedio de días de vida al momento del diagnóstico fue de $17 \pm 10$ días. El método de diagnóstico más utilizado fue la Inmunofluorescencia indirecta. Los aislamientos virales más frecuentes fueron: el virus sincitial respiratorio, virus de la Influenza A y B. Se encontró una elevada morbilidad respiratoria ya que más de la mitad necesitaron soporte respiratorio y poco más de un cuarto recibieron antibióticos. No se registró muerte atribuidas a la infección viral. 


\section{REFERENCIAS BIBLIOGRÁFICAS}

1. Pichler $K$, Assadian O, Berger A. Viral Respiratory Infections in the Neonatal Intensive Care Unit: A review. Front Microbiol. 2018; 9(October). DOI: https://doi.org/10.3389/fmicb.2018.02484

2. Lanaspa M, Annamalay AA, Lesouëf P, Bassat Q. Epidemiology, etiology, x-ray features, importance of coinfections and clinical features of viral pneumonia in developing countries. Expert Rev Anti Infect Ther. 2014; 12(1):31-47. https://doi.org/10.1586/14787210.2014.866517

3. Calvo C, Garcia M, Casas I, Perez Breña P. Infecciones respiratorias virales. En Protocolos diagnósticoterapéuticos de la AEP: Infectología pediátrica. AEP; 2011. p. 189-204.

4. Lambkin-williams R, Noulin N, Mann A, Catchpole A, Gilbert AS. The human viral challenge model: accelerating the evaluation of respiratory antivirals, vaccines and novel diagnostics. Respir Res. 2018; 19(123):1-15.

5. Sonego M, Pellegrin MC, Becker G, Lazzerini M. Risk factors for mortality from acute lower respiratory infections (ALRI) in children under five years of age in low and middle-income countries: A systematic review and meta-analysis of observational studies. PLoS One. 2015; 10(1):1-17.

6. Luoto R, Jartti T, Ruuskanen O, Waris M, Lehtonen L, Heikkinen T. Review of the clinical significance of respiratory virus infections in newborn infants. Acta Paediatr. 2016; 105(10):1132-9.

7. Townsi N, Laing IA, Hall GL, Simpson SJ. The impact of respiratory viruses on lung health after preterm birth. Eur Clin Respir J. 2018;(5):1-11.

8. Doan Q, Enarson P, Kissoon N, Klassen TP JD. Rapid viral diagnosis for acute febrile respiratory illness in children in the Emergency Department. Cochrane Database Syst Rev. 2014; (9):1-55.

9. Gonzalez-Carrasco E, Calvo C, Garcia-Garcia ML, Beato M, Munoz-Archidona C, Pozo F, et al. Infecciones virales de las vias respiratorias en la Unidad de Cuidados Intensivos Neonatales. An Pediatr. 2015; 82(4):242-6.

10. Ronchi A, Michelow IC, Chapin KC, Bliss JM, Pugni L, Mosca F, et al. Viral respiratory tract infections in the neonatal intensive care unit: The virion-i study. J Pediatr. 2014; 165(4):690-6.

11. Bennett NJ, Tabarani CM, Bartholoma NM, Wang D, Huang D, Riddell SW, et al. Unrecognized viral respiratory tract infections in premature infants during their birth hospitalization: A prospective surveillance study in two neonatal intensive care units. J Pediatr. 2012; 161(5):814-818.

12. Drysdale SB, Wilson T, Alcazar M, Broughton S, Zuckerman M, Smith M, et al. Lung function prior to viral lower respiratory tract infections in prematurely born infants. Thorax. 2011;66(6):468-73.

13. Drysdale SB, Alcazar M, Wilson T, Smith M, Zuckerman M, Lauinger IL, et al. Respiratory outcome of prematurely born infants following human rhinovirus A and Cinfections. Eur J Pediatr. 2014; 173(7):913-9.

14. Forsey JT, Elmasry OA, Martin RP. Patent arterial duct. Orphanet J Rare Dis. 2009; 4:1-9.

15. Verboon-Maciolek MA, Krediet TG, Gerards LJ, Fleer A, Van Loon TM. Clinical and epidemiologic characteristics of viral infections in a neonatal intensive care unit during a 12-year period. Pediatr Infect Dis J. 2005; 24(10):901-4.

16. Higdon MM, Le T, O'Brien KL, Murdoch DR, Prosperi $\mathrm{C}$, Baggett $\mathrm{HC}$, et al. Association of C-reactive protein with bacterial and respiratory syncytial virus-associated pneumonia among children aged $<5$ years in the PERCH study. Clin Infect Dis. 2017; 64(3):378-86.

17. Resch B, Michel-Behnke I. Respiratory syncytial virus infections in infants and children with congenital heart disease. Curr Opin Cardiol. 2013; 28(2):85-91.

18. Pedersen J, Hedegaard ER, Simonsen U, Krüger M, Infanger M, Grimm D. Current and Future Treatments for Persistent Pulmonary Hypertension in the Newborn. Basic Clin Pharmacol Toxicol. 2018; 123(4):392-406.

19. Siegel JD, Rhinehart E, Jackson M CL. 2007 Guideline for isolation precautions: Preventing Transmission of Infectious Agents in Healthcare Settings. Centers Dis Control Prev. 2018; 35(10):1-204.

20. Cerone JB, Santos RP, Tristram D, Lamson DM, Stellrecht KA, St George K, et al. Incidence of respiratory viral infection in infants with respiratory symptoms evaluated for late-onset sepsis. J Perinatol. 2017; 37(8):922-6.

21. Pados BF. Physiology of Stress and Use of Skin-to-Skin Care as a Stress-Reducing Intervention in the NICU. Nurs Womens Health. 2019;23(1):59-70.

22. Mammas IN, Greenough A, Theodoridou M, Kramvis A, Rusan M, Melidou A, et al. Paediatric Virology and its interaction between basic science and clinical practice. Int J Mol Med. 2018;41(3):1165-76. 